\title{
Spontaneous magnetization reversal caused by magnetic noise in $\varepsilon-\mathrm{In}_{0.24} \mathrm{Fe}_{1.76} \mathrm{O}_{3}$ nanoparticles
}

\author{
Alexei Dmitriev* \\ Institute of Problems of Chemical Physics, Russian Academy of Sciences, 142432 Chernogolovka, Russia
}

\begin{abstract}
Kinetics of magnetization relaxation of the exotic $\varepsilon-\operatorname{In} 0_{.24} \mathrm{Fe}_{1.76} \mathrm{O}_{3}$ nanoparticles under applied magnetic field has been studied. The fluctuation field and the activation volume have been calculated from the magnetic viscosity data. The relation between magnetic viscosity and magnetic noise caused by the random thermally activated magnetization reversal of a single nanoparticle has been established. Stepped sweeping of magnetic field expands the windows of experimentally detectable magnetic fluctuations. The changes in the reversal magnetic field provide $\varepsilon-\mathrm{In}_{0.24} \mathrm{Fe}_{1.76} \mathrm{O}_{3}$ nanoparticles scanning and sorting them by magnetic noise frequency.
\end{abstract}

\section{Introduction}

Recently it has become possible to create new nanomagnets based on the epsilon phase of iron (III) oxide $\varepsilon-\mathrm{Fe}_{2} \mathrm{O}_{3}$ with a giant (up to $2.34 \mathrm{~T}$ ) coercive force [1]. This exceeds all known values revealed by such magnets as $\mathrm{Fe}_{14} \mathrm{Nd}_{2} \mathrm{~B}$ or $\mathrm{SmCo}_{5}$. Permanent magnets based on $\varepsilon-\mathrm{Fe}_{2} \mathrm{O}_{3}$ nanoparticles are much cheaper than rare-earth magnets such as $\mathrm{Nd}_{14} \mathrm{Fe}_{2} \mathrm{~B}$ and $\mathrm{SmCo}_{5}$. Static magnetic properties of $\varepsilon-\mathrm{Fe}_{2} \mathrm{O}_{3}$ nanoparticle ensembles were studied in detail [2]. Time stability of parameters of permanent magnets is one of the main criteria of applicability of materials in various devices. Therefore, in this work, slow relaxation of magnetization of the epsilon phase of iron oxide nanoparticle ensembles has been analyzed.

Slow magnetization reversal (magnetic viscosity) in different magnets is caused by magnetic noise [3]. Despite intensive investigations of $\varepsilon-\mathrm{Fe}_{2} \mathrm{O}_{3}$ nanomagnets, the problems of the spectrum of magnetic fluctuations, spontaneous demagnetization mechanisms and time stability are still open questions. The purpose of this work is to find a relation between magnetic viscosity and spectral characteristics of magnetic fluctuations in epsilon phase of iron oxide nanoparticle ensembles.

\section{Experimental}

The epsilon phase of iron (III) oxide $\varepsilon$ - $\operatorname{In}_{0.24} \mathrm{Fe}_{1.76} \mathrm{O}_{3}$ nanoparticles with a length of $80 \mathrm{~nm}$ and a diameter of $35 \mathrm{~nm}$ (Fig. 1) were fabricated by two methods, namely, synthesis in inverse micelles and sol-gel method. The nanoparticles have orthorhombic crystal structure with four non-equivalent cationic positions of $\mathrm{Fe}^{3+}$ ions. One of them has tetrahedral and three others octahedral environment. Indium ions are substitution impurities in the octahedral positions of iron ions. Synthesis and characterization of the nanowires were described in detail in $[4,5]$.

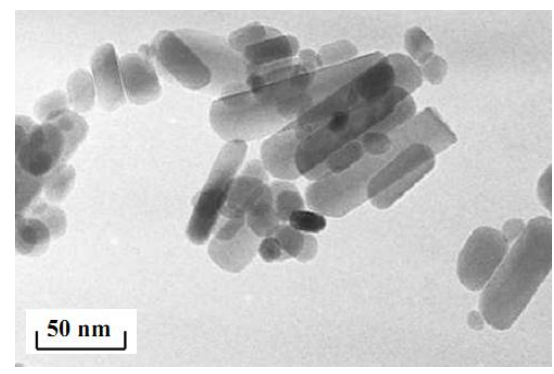

Fig. 1. TEM image of $\varepsilon-\operatorname{In}_{0.24} \mathrm{Fe}_{1.76} \mathrm{O}_{3}$ nanoparticles.

A Quantum Design MPMS 5XL SQUID magnetometer was used in standard measurements of the field dependence $M(H)$ of sample magnetic moment. The SQUID magnetometer was also used to obtain time $M(t)$ dependences of magnetic moment for magnetic fields in the range $H=1-13 \mathrm{kOe}$ at $T=300 \mathrm{~K}$. Fig. 2(a) shows the scheme of applied magnetic field switching in measurements of the $M(t)$ dependences.

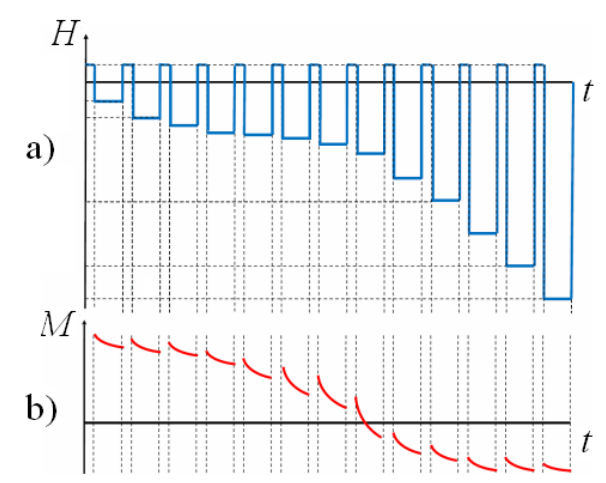

Fig. 2. Scheme of applied magnetic field switching in the experiments. 
A sample was first magnetized to saturation in magnetic field $H=50 \mathrm{kOe}$, which is higher than the saturation field. Then the field directed opposite to the magnetization of the sample was turned on. After negative magnetic field became steady, the time dependence of magnetization of a sample was recorded. Fig. 2(b) shows the schematic diagram of relaxation of the magnetic moment of a sample.

\section{Results and discussion}

\subsection{Magnetic viscosity}

The time dependence of magnetic moment increment $\Delta M(t)$ recorded in the reverse magnetic field $H=9 \mathrm{kOe}$ at $T=300 \mathrm{~K}$ is shown in normal and semi-logarithmic coordinates in Fig. 3.

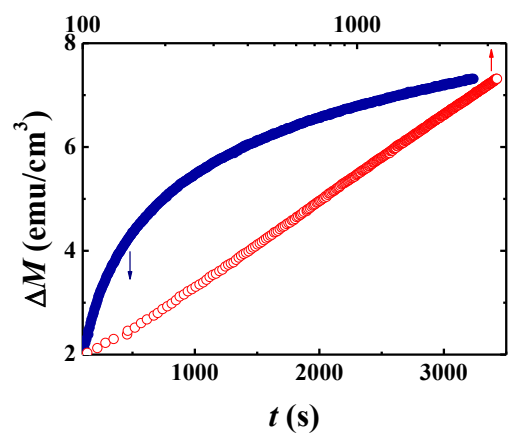

Fig. 3. Time dependence of magnetic moment $\Delta M(t)$ at $T=300$ $\mathrm{K}$ in external magnetic field $H=9 \mathrm{kOe}$ in normal and semilogarithmic coordinates.

The dependence $\Delta M(t)$ follows the logarithmic law:

$$
\Delta M \sim S \ln t
$$

Tangent of the slope of $\Delta M(\ln t)$ curve corresponds to magnetic viscosity $S$ :

$$
S=k_{\mathrm{B}} T / \Delta E
$$

where $k_{\mathrm{B}}$ is the Boltzmann constant, $T$ - temperature, $\Delta E$ - range of activation energy of magnetic fluctuations.

Time dependences $\Delta M(t)$ were recorded in different magnetic fields at $T=300 \mathrm{~K}$ (Fig. 4).

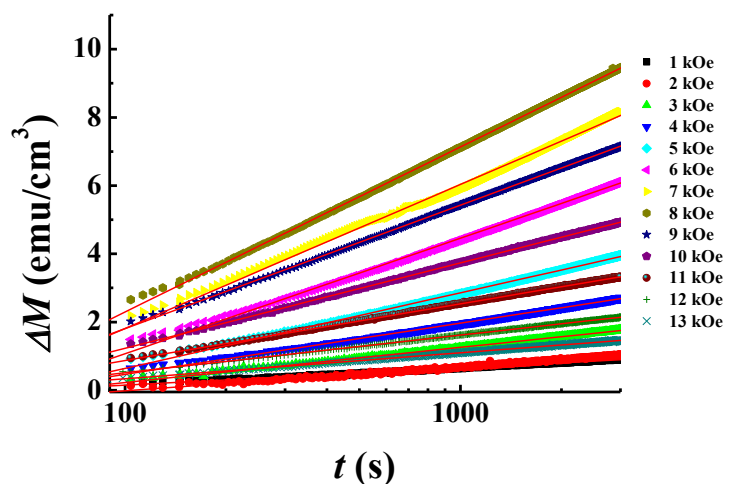

Fig. 4. Time dependences of magnetic moment $\Delta M(t)$ at $T=$ $300 \mathrm{~K}$ in different magnetic fields. Solid lines show approximations.
Slopes of these curves and correspondent magnetic viscosities are non-monotonic functions of magnetic field (Fig. 5).

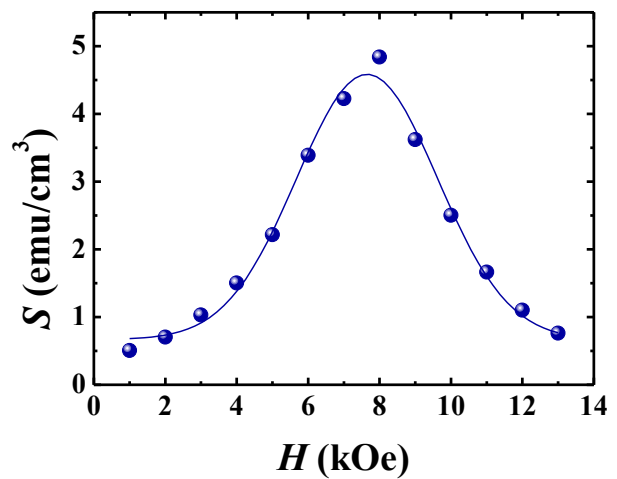

Fig. 5. Field dependence of viscosity $S$ at $T=300 \mathrm{~K}$. Solid line is a guide for the eye.

Field dependences $S(H)$ at $T=300 \mathrm{~K}$ show maximum corresponding to $8 \mathrm{kOe}$. The shape of the $S(H)$ depends on volume distribution of nanoparticles $f\left(V_{C}\right)$. Those nanoparticles contribute to magnetic relaxation only, which volume is equal to critical nanoparticle volume $V_{\mathrm{C}}$ $=25 k_{\mathrm{B}} T /\left(K\left(1-\left(H / H_{\mathrm{a}}\right)^{2}\right)\right)$. Here, $K$ is constant of magnetic anisotropy, $k_{\mathrm{B}}$ is Boltzmann constant, $H_{\mathrm{a}}$ is magnetic anisotropy field. Relaxation of nanoparticles of smaller volume $V<V_{\mathrm{C}}$ is finished when new value of magnetic field is attained, i.e. before time dependence recording. The nanoparticles of larger volume $V>V_{\mathrm{C}}$ do not contribute to magnetic relaxation because their energy barrier separating opposite directions of magnetization is too high in comparison with the energy of thermal fluctuations. In case of lognormal distribution $f\left(V_{C}\right)$ shape of the $S(H)$ dependence is close to the lognormal distribution function. The peak position of $S(H)$ dependence corresponds to the magnetization reversal of the nanoparticles coinciding to the mode of distribution $f\left(V_{C}\right)$.

\subsection{Fluctuation field and activation volume}

Fluctuation field $H_{\mathrm{f}}$ is another fundamental parameter additionally to magnetic viscosity $S$ [6]. The fluctuation field was used to compare magnetic fluctuations intensity with typical parameters of the nanoparticles expressed in the same units (anisotropy field, coercive field, etc). Fuctuation field is defined by equation [7]:

$$
H_{\mathrm{f}}=S / \chi_{\text {irr }}
$$

where $\chi_{\text {irr }}$ is irreversible susceptibility expressed as

$$
\chi_{\text {irr }}=\chi /(1+N \chi)
$$

where $N=2 \pi$ is the demagnetizing factor of cylinder and $\chi=\mathrm{d} M / \mathrm{d} H$ is the slope of tangent to demagnetizing curve of magnetic hysteresis loop [8,9] (Fig. 6). Field dependence $\chi_{\text {irr }}(H)$ at $T=300 \mathrm{~K}$ show maximum corresponding to $7 \mathrm{kOe}$ (fig. 7). Positions of the $S(H)$ and $\chi_{\text {irr }}(H)$ maxima are close to coercive field $H_{\mathrm{c}}=6 \mathrm{kOe}$ determined from the hysteresis loop [8, 9] (Fig. 6). 


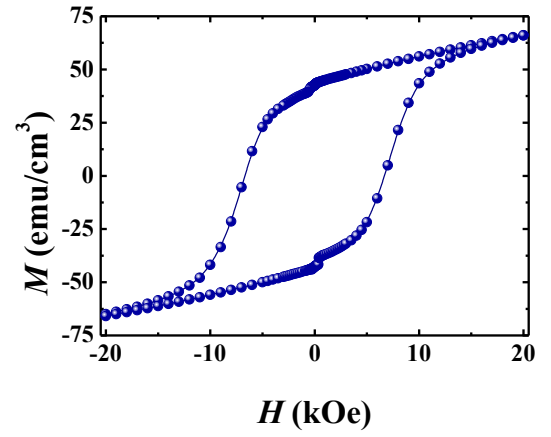

Fig. 6. Magnetic hysteresis loop in $\varepsilon-\mathrm{In}_{0.24} \mathrm{Fe}_{1.76} \mathrm{O}_{3}$ nanoparticles at $T=300 \mathrm{~K}$. Solid line is a guide for the eye.

A small discrepancy is caused by measurement uncertainty of magnetic field, defined as half of the step of the magnetic field sweep, which is $1 \mathrm{kOe}$.

The average fluctuation field $H_{\mathrm{f}}=315$ Oe at $T=300$ $\mathrm{K}$ was estimated from Eqs. (3) and (4). The $H_{\mathrm{f}}$ value is close to typical values in hard magnetic materials such as $\mathrm{Nd}_{2} \mathrm{Fe}_{14} \mathrm{~B}[10]$.

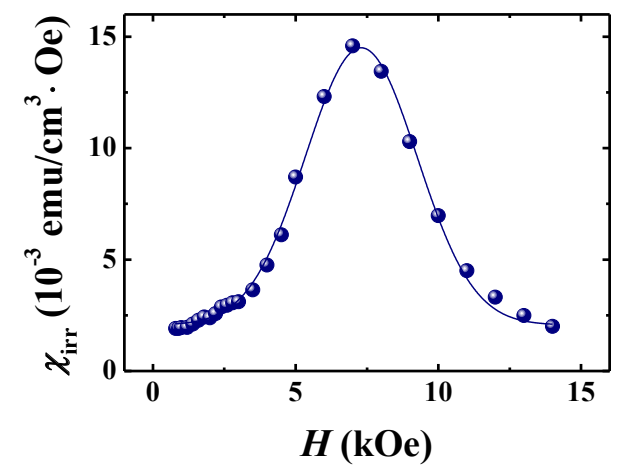

Fig. 7. Field dependence of irreversible magnetic susceptibility $\chi_{\text {irr }}$ at $T=300 \mathrm{~K}$. Solid line is a guide for the eye.

The $H_{\mathrm{f}}$ value can be used to calculate activation volume [8]:

$$
V_{\mathrm{a}}=k_{\mathrm{B}} T / M_{\mathrm{S}} H_{\mathrm{f}}
$$

$M_{\mathrm{s}}=84.4 \mathrm{emu} / \mathrm{cm}^{3}$ at $T=300 \mathrm{~K}$ [7]. The activation volume corresponds to the average volume of thermal activation. Average activation volume $V_{\mathrm{a}}=1.6 \cdot 10^{-18} \mathrm{~cm}^{3}$ at $T=300 \mathrm{~K}$ was estimated from Eq. (5). The $V_{\text {a value is }}$ noticeably smaller than the volume of the single $\varepsilon$ $\mathrm{In}_{0.24} \mathrm{Fe}_{1.76} \mathrm{O}_{3}$ nanoparticle. This implies that only a small part of its volume is involved in the elementary act of magnetization reversal.

\subsection{Spectral density of magnetic fluctuations}

On the assumption of a relaxation time distribution $F(\tau)$, spectral density of magnetic fluctuations $W(f)$ is defined by the following equation [3]:

$$
W=k_{\mathrm{B}} T / 2 f \Delta E
$$

When comparing Eqs. (2) and (6), we can find a relation between magnetic viscosity and spectral density of thermal fluctuations:

$$
W(f)=S / 2 f
$$

Equation (7) is a "bridge" connecting the macroscopic phenomenon of magnetic viscosity and the microscopic fluctuation phenomena of magnetization reversal of a single nanoparticle. Thus, when controlling magnetic viscosity by changing magnetic field, we change the width of "window" for detecting a spectrum of magnetic noise.

\section{Conclusion}

Time dependences of magnetization reversal of $\varepsilon$ $\mathrm{In}_{0.24} \mathrm{Fe}_{1.76} \mathrm{O}_{3}$ nanoparticles in the reverse magnetic field were measured and analyzed in a wide range of magnetic field strength. Logarithmic kinetics of magnetization reversal allowed field dependences of magnetic viscosity to be extracted. The logarithmic kinetics of magnetic viscosity is determined by a superposition of thermally activated magnetization reversal of a single nanoparticle with a wide relaxation time distribution. A relation between magnetic viscosity and spectral characteristics of magnetic fluctuations in nanoparticle ensembles was found. The fluctuation field and the activation volume were calculated from the magnetic viscosity data.

Supported by RFBR, project no. 16-07-00863 a. Author is grateful to Prof. R.B. Morgunov for samples providing, measurements support and discussion.

\section{References}

1. J. Jin, S. Ohkoshi, K. Hashimoto, Adv. Mater. 16, 48 (2004).

2. J. Tucek, R. Zboril, A. Namai, S. Ohkoshi, Chem. Mater. 22, 6483 (2010).

3. A.I. Dmitriev, A.D. Talantsev, E.I. Kunitsyna, R.B. Morgunov, V.P. Piskorskii, O.G. Ospennikova, E.N. Kablov, J. Exp. Theor. Phys. 123, 303 (2016).

4. S. Sakurai, S. Kuroki, H. Tokoro, K. Hashimoto, S. Ohkoshi, Adv. Funct. Mater. 17, 2278 (2007).

5. K. Yamada, H. Tokoro, M. Yoshikiyo, T. Yorinaga, A. Namai, S. Ohkoshi. J. Appl. Phys. 111, $07 B 506$ (2012).

6. L. Neel, J. Phys. Radium 11, 49 (1950).

7. E.P. Wohlfarth, J. Phys. F: Met. Phys. 14, L155 (1984).

8. A.I. Dmitriev, O.V. Koplak, A. Namai, H. Tokoro, S. Ohkoshi, R.B. Morgunov, Phys. Solid State 55, 2252 (2013).

9. A.I. Dmitriev, O.V. Kolpak, A. Namai, H. Tokoro, S. Ohkoshi, R.B. Morgunov, Phys. Solid State 56, 1795 (2014).

10. J.C. Martinez, F.P. Missell, J. Appl. Phys. 64, 5726 (1988). 